\title{
Concise Commentary: Visceral Obesity, Sarcopenia, and Cancer Surgery-Increasing Fitness Decreases Risk
}

\author{
Oliver A. Varban ${ }^{1}$
}

Published online: 16 April 2018

○) Springer Science+Business Media, LLC, part of Springer Nature 2018

\begin{abstract}
Although obesity, already recognized as a worldwide epidemic, is associated with multiple comorbid conditions, one of its most underrated effects is its impact on the resource utilization and complications accompanying surgery $[1$, 2]. Likewise, sarcopenia is considered as one of the most important causes of functional decline and adverse health outcomes, particularly in the elderly [3, 4]. In this issue of Digestive Diseases and Sciences, Chen et al. [5] perfectly demonstrate the impact of sarcopenia, visceral obesity, and age on the surgical management of colorectal cancer, reporting an increased risk of postoperative complications. The authors, in a prospective study, used objective metrics to define visceral obesity and sarcopenia via cross-sectional computed tomography (CT), conditions associated with increased mortality after major elective abdominal surgery [6]. In addition to CT imaging, the authors also used muscle strength and physical performance to further characterize sarcopenia. In doing this, the authors gathered important data necessary to meet their objective, which was to ascertain the combined impact of visceral obesity and sarcopenia on short-term outcomes in patients undergoing colorectal surgery for cancer.
\end{abstract}

The authors noted that one-third of their patients were defined as having sarcopenia and that one-half of their patients had visceral obesity, not inconsequential numbers. The combination of sarcopenia and visceral obesity was an independent risk factor for total, surgical, and medical complications as well as increased hospital length-of-stay and cost. Interestingly, the use of laparoscopic-assisted surgical techniques was protective, indicating the importance of minimally invasive techniques among patients with obesity

Oliver A. Varban

ovarban@med.umich.edu

1 Department of Surgery, Michigan Medicine, 2926

Taubman Center, 1500 E Medical Center Drive, SPC 5343,

Ann Arbor, MI 48109-5343, USA and sarcopenia, although it remains to be seen if this is the case with normal-risk patients.

Even though the authors identify obesity and sarcopenia as risk factors identified prior to colorectal cancer surgery, optimizing these risk factors preoperatively can be challenging, particularly in cancer patients when time is limited due to disease progression. Although the authors did not define the interval of time between cancer diagnosis and surgery, this time can be crucial to improving outcomes. Interventions for improving obesity and sarcopenia in the preoperative setting are currently not widely adopted. The most effective treatment for obesity is bariatric surgery, though given the multiple barriers to care as well as the amount of time need to maximize weight loss (i.e., 1 year), it is not a feasible intervention in the setting of cancer, unless it is offered to high-risk patients in the years before the diagnosis is made. "Pre-habilitation," which includes exercise therapy and nutritional supplementation prior to surgery, has demonstrated beneficial effects on surgical patients [7]. In Michigan, a home-based, preoperative training program (mean duration of 34 days) was associated with a $31 \%$ reduction in hospital duration of stay and $28 \%$ lower total costs among patient undergoing major elective general and thoracic procedures, giving impetus for adopting the program more broadly. Thus, future studies on improving postoperative outcomes among patients with obesity and sarcopenia should also incorporate the impact of pre-habilitation programs, which can be initiated at the time of CT imaging. Given the time constraints in pursuing surgical management of cancer, it remains to be seen if obesity and sarcopenia can be treated at the " 11 th hour."

Acknowledgments Salary support from Blue Cross Blue Shield of Michigan for participation and leadership in collaborative quality improvident initiatives. 


\section{References}

1. Hawn MT, Bian J, Leeth RR, et al. Impact of obesity on resource utilization for general surgical procedures. Ann Surg. 2005;241:821-826. (discussion 826).

2. Merkow RP, Bilimoria KY, McCarter MD, Bentrem DJ. Effect of body mass index on short-term outcomes after colectomy for cancer. J Am Coll Surg. 2009;208:53-61.

3. Kim TN, Choi KM. Sarcopenia: definition, epidemiology, and pathophysiology. J Bone Metab. 2013;20:1-10.

4. Sheetz KH, Waits SA, Terjimanian MN, et al. Cost of major surgery in the sarcopenic patient. J Am Coll Surg. 2013;217:813-818.
5. Chen, W, Chen X, Ma L, et al. Impact of visceral obesity and sarcopenia on short-term outcomes after colorectal cancer surgery. Dig Dis Sci. (Epub ahead of print). https://doi.org/10.1007/s1062 0-018-5019-2.

6. Englesbe MJ, Lee JS, He K, et al. Analytic morphomics, core muscle size, and surgical outcomes. Ann Surg. 2012;256:255-261.

7. Englesbe MJ, Grenda DR, Sullivan JA, et al. The Michigan surgical home and optimization program is a scalable model to improve care and reduce costs. Surgery. 2017;161:1659-1666. 